\title{
Chinese Philosophy of Harmony in the Use of Logo Designing
}

\author{
Xinxiong Liu \\ Huazhong University of Science and Technology \\ Wuhan, Hubei, P. R. China 430074
}

\author{
Wenqi Cao \\ Huazhong University of Science and Technology \\ Wuhan, Hubei, P. R. China 430074
}

\author{
Qian Ji \\ Huazhong University of Science and Technology \\ Wuhan, Hubei, P. R. China 430074
}

\begin{abstract}
The various factions in Chinese intellectual history, especially the three pillars of the Chinese philosophy of Confucianism, Taoism, Buddhism, are invariably expressed the hope and longing towards "he".Today, in the pursuit of a socialist harmonious society, the research on traditional philosophy of harmonious theory undoubtedly has an important inspiration and reference. Harmony theory not only is the guiding philosophy of today's social development, but also the guidance of enterprise under the social environment. Logo is a product of the modern economy, it is different from the imprint of the ancient time. "He xie"(harmony) is necessity for society, and it is also desire of the people. Signs should also be guided by harmonious philosophy. Designers should also seek to breakthrough from the rich and deep Chinese philosophy of harmony, and give profound meanings to the logo designs. This paper tries to further study the use of Chinese philosophy of harmony in logo design, hoping to find a meeting point of logo design and Chinese philosophy of harmony and provide some useful theoretical basis for the further development of logo designing.
\end{abstract}

Keywords-logo design; Chinese philosophy; harmony; traditional culture

\section{INTRODUCTION}

In Chinese culture, "he" and "xie" are synonymous, and "he xie"(harmony) appears as the meaning of "he" in the ancient time. The various factions in Chinese intellectual history, especially the three pillars of the Chinese philosophy of Confucianism, Taoism, Buddhism, are invariably expressed the hope and longing towards "he".

"He" was first conceived in the ancient rituals of witchcraft. This primitive early human cultural pattern was gradually divided into "yue" and "li". "Yue", in ancient times, refers to a trinity of music, song (poem) and dance, Chinese classical aesthetics attaches great importance to "yue" contained and embodied "he". Compared to the ancient Greek aesthetics, Qin aesthetics believes that the beauty of "he" lies not only in the unity of form of nature, but also lies in the major ethical and moral implications shown in this unity, this thoughts are obviously far more richer and deeper.
"He xie"(harmony) is also closely associated with the generating and development of "ying yang wu xing"(the five elements of ying and yang). "Wu xing"(the five elements) have early origin, they were first described by Jizi, as "water, fire, wood, metal and earth", was actually the most essential and direct five substances to sustain basic human survival. "Yi zhuan" see "yin" and "yang" as two basic concepts explaining for "bagua", thus explain everything. "Yi jing", by presenting different meanings of names of "gua" and 64 kinds of different orders of the guas, reflects the opposition of yin and yang as well as the concept of coordination, unity and harmony, as their foundation and heritage; and "yi zhuan" on a macro philosophical level, summarized and revealed the intrinsic order of the universe: "roads changes, the positive life, maintaining harmony, thus beneficial", and summarized the harmony of the universe as "a perfect combination of yin and a yang".

"Heaven and human are one" is one of the fundamental concepts of classical Chinese philosophy, and "Heaven and human are deviated" announced the opposite. Since the establishment of rational spirit in Qin, "God and human are one" gradually gave up to "Nature and human are one" which believes that man are in harmony with nature and society. Dong Zhongshu ethicized natural things, personified the nature of heaven, grant "three cardinal guides" of the position fixed by heaven, while, with the authority of the heaven, curb the absolute power of king, emphasizing the importance of associating the social order to the laws of nature to exist as a harmonious and stable overall. On this basis, Dong Zhongshu elevated "he" to a height of philosophical ontology: "He is produced by heaven and earth", "the beauty of heaven and earth is no bigger than".

"He" is the wisdom of life which is summed up by classical Chinese philosophy in the inquiry of the relationships between "heaven and human", "human and human" and "human with me". Confucianism, Taoism, Buddhism, the three pillars of Chinese philosophy, have developed different ideas on "he". "He" has a special status in the Confucian ideology. The starting point of Confucian is a individual-group relations and individual-me relations, pursuing harmony between people. 
"Tao", with a major trait of "he", is the core of Taoism. Buddhism's starting point is a reason-desire relations and physical-psychological relations, pursuing of harmony within human body. Today, in the pursuit of a socialist harmonious society, the research on traditional philosophy of harmonious theory undoubtedly has an important inspiration and reference.

\section{HARMONIOUS BEAUTY IN LOGO}

There is a pursuit of harmony in modern society, a socialist harmonious society is the strategic goal of China's social development, it refers to a social status of friendly, harmonious and concerted efforts of all sectors. Harmonious theory has a long history, rich heritage and profound meaning in China, it is the essence of Chinese culture, the inexhaustible resource of the endless generate of Chinese nation.

Early logo design is simple, by doing logo directly with a board or plaque, plain and simple. With the progress of society, companies need the logo to carry more meanings and deliver more company information to the user. Harmony theory not only is the guiding philosophy of today's social development, but also the guidance of enterprise under the social environment. Logo is a product of the modern economy, it is different from the imprint of the ancient time. Modern logo, bearing the company's intangible assets, is a delivery media of comprehensive company information. From the formulation of national development strategies to macro-micro social interaction between people; from the international cultural communication to the local culture blend; from company culture to the logo design, we can see that "He xie"(harmony) is necessity for society, and it is also desire of the people. Signs should also be guided by harmonious philosophy. Designers should also seek to breakthrough from the rich and deep Chinese philosophy of harmony, and give profound meanings to the logo designs.

Philosophy theory is the heart and soul of the culture of a nation, it is the summed-up experience of people transforming and conquering nature, in the mean while, it also has a methodology guiding value to people's next action. This paper tries to further study the use of Chinese philosophy of harmony in logo design, hoping to find a meeting point of logo design and Chinese philosophy of harmony and provide some useful theoretical basis for the further development of logo designing.

\section{THE EXEMPLIFICATION OF HARMONIOUS THEORY IN LOGO DESIGNING}

\section{A. Harmony between Human and Nature}

Confucianism advocates "Heaven and human are one". That is to say, to see the unity of Heaven in the unity of humanity, communicate and coordinate heaven and human by the bridge of humanity, emphasizing on harmony and unity between subject and object. It believes that everything in the world is a whole in harmony and unity, and has followed the same rule of nature. It sees the heaven, earth, human, art and morality as a vibrant organic whole, it gives human emotion the form of objects, by using various approaches such as contrast, it uses objects to express emotions, in order to achieve the effect of "be vivid by expressing the meanings by shape". Under the effect and the mode of this aesthetic idea, forms the uniqueness of Chinese visual arts expressions of "less reality, more meanings", "less reappearance, more performance", focus on the overall shape of the momentum of the object, rather than staying on the subtle level of depicting it.

The logo of 2011 Expo Park designed by the designer Mr. Chen Shaohua, use the concept of the Expo Park theme-"Heaven Chang'an, creative nature", as the core, referring to the sentence from the Tang poem, "Pleased with the vernal breeze and my swift trotting steed; Ere the day's done, we've all the Chang'an flowers seen". The idea comes from the "Tao Te Ching": "Tao gave birth to the One; The One gave birth successively to two things, Three things, up to ten thousand", and it constructed the idea by using the abstract aesthetic and figurative innovation of modern design, got the original idea from "the shape of flowers", which is the essence of the nature. It gathered the original shape of hundreds of flowers and simplifies the synthesis into a new shape, by combining triangular, quadrilateral, pentagonal, hexagonal and many types of flowers shapes, into "Flowers Lucky Pattern", one mother pattern full of oriental charm: Dawson one, one two, two three, three things, the use of modern design aesthetic and representational abstract construct innovative ideas to the essence of nature's pattern for the original ideas its original state CASINO flowers, simplification synthesize new shape, the triangle, quadrilateral, pentagonal, hexagonal many types of flowers, combined into one full of oriental charm of the Hundred Flowers Kat India, the mother-shaped pattern: three things, flowers auspicious; Tetraena as soil, hemispherical dome; five leaves raw wood, Linsen shadow education; six streams into water, bring benefit to people. It also symbolizes a few simple geometric shapes people, city, community, environment in one, meaning flower, soil, grass, wood, water symbiosis. These few simple geometric shapes also symbolizes people, city, community, the environment are in one, meaning that flowers, soil, grass, wood, water symbiosis. "Fig. 1"
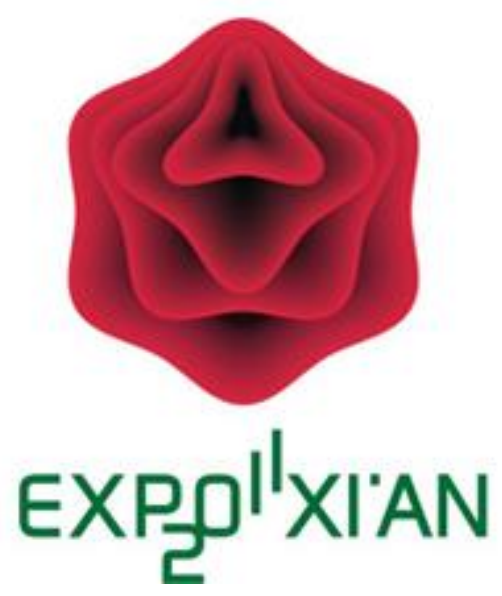

Fig. 1. Xi'an Expo Park Logo

\section{B. Harmony of Graphic Layout}

1) Beauty of Balance: According "Zhong Yong", when dealing with affairs, Emperor Shun use the way of "dominate both ends, apply the middle on people". "Zhi liang yonng 
zhong"(dominate both ends, apply the middle on people) means when dealing with things, we must grasp the contradiction of opposites of "both ends", only in this way, can we get the "middle", so as to better use the "middle", so that we would be able to meet the general rule and handle things impartially. Thus, ritual and benevolence can achieve harmonious unity and perfect implement, society can be run orderly, we can achieve "one world" of ease and beauty. The influence of the idea of "Zhi liang yonng zhong" reflected on ancient Chinese architecture, is that the layout of the building has a preference to be on the central axis, putting the major construction or arrangement on the central axis line.

Confucius aspire for a better society which is "the world with its rule" and "He", which is a highly harmony between human and society, he cited "Shi Jing" once described: "He was neither violent nor remise, neither hard nor soft. Gently he spread his instructions abroad, and all dignities and riches were concentrated in him. That has reference to the perfection of such harmony.". It means all moderately coordinated that is just right. Zhuxi had made this explanation to the "zhong"(middle) of "zhong yong"(moderation):" 'zhong' is the truth that is just right." "Li Ji • Zhong Yong" says "The superior man's embodying the course of the Mean is because he is a superior man, and so always maintains the Mean". So moderation is not rigid, mechanical, static, but flexible, dynamic and changing . Moderation is in tone of harmony, is a approach of dialectical unity with the goal to pursue the overall harmony. "The moon full, then start missing", "water full, then overflows ", it indicates that there is a matter of degree in development of things, things would only achieve harmony in the range of degree. The overall harmony is the most essential feature of moderation, moderation opposes excess and scarcity, it advocates impartiality and refuses extremes, thus to keep things balanced and coordinated. It believes that by "holding the moderation", "he"(harmony) can be achieved, that is the best overall harmonious situation. This concept is fully reflected by the logo of Bank of China designed by Mr. Kan Taiqiang in 1986. Use the knot coin as the basic starting point of the design, combines the structure of the Chinese character " 中"(first word of China), perfectly embodies the company name and banking business characteristics. We can understand the essence of the moderation as of the wisdom of seeking balance. "Fig. 2"

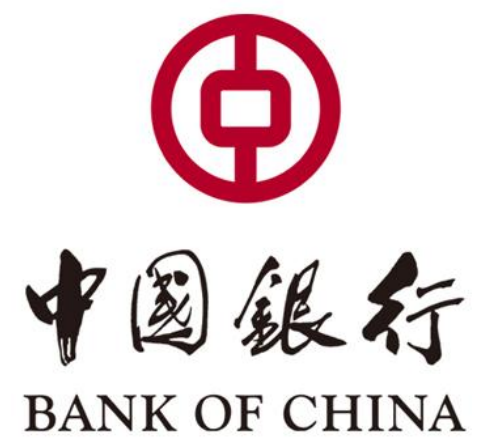

Fig. 2. The Logo of Bank of China
2) Beauty of Vividness: Laozi thinks everything in the world was founded by the yin and yang, solid and obscure. The appearance, changes and developments of everything in the world are specific rules of nature. The birth and death of things, solid or obscure, there is a pattern. Chinese calligraphy which is most typically affected by this concept, not only pursue the "solidity" of the gestures of vigorous, power and brilliant, but also pay attention to the blank left between strokes.

Taiji pattern with the white black beauty is portrayal of beauty of vividness, Taiji is a cohesion and an interaction of yin and yang, these two parts endlessly rotate around a circle. This is an dynamic pattern of obscurity and solidity go hand in hand, fullness and emptiness rely on each other, contrary movement complete one another. Taiji Pattern originally expressed the ancients' simple understanding to the nature of universe, it has a strong dialectical trait. It bears all dialecticalunited relations including heaven and earth, sun and moon, male and female, up and down, etc. It indicates that everything in the world is in movement, change and development, due to the contradiction between the yin and yang of dialecticalunited. "Zhouyi • Xicishang" says: "yin and yang is the rule". Taiji pattern of yin and yang is embodied by black and white, black represent yin, white represent yang. This black and white form a mutual aid from each other to set off their own, as long as one part has changed, it would also cause the change of the other, thus forms a kind of interact and dependent relations, by the words of modern design, it forms the relations of positivenegative shaping. Guiding by the shape of $S$, the viewer's sight would waver between black and white and rotate endlessly.

"Dancing Beijing", the logo of 2008 Beijing Olympic Games is a manifestation of it. The logo is composed of three parts: 1.a Chinese pattern of a man-shape character "Jing"; 2. pinyin of the word "Beijing" and "2008", symbolizing 2008 Beijing Olympic Games; 3. Olympic rings: symbol of Olympic spirit. The pattern looks like the "Jing" in Beijing, and a dancing humanoid, chic and elegant, full of tension. The new logo is a collection of traditional Chinese seal, calligraphy and other art forms and movement characteristics, making an unique combination of Chinese spirit, Chinese charm and Chinese culture, symbols an open, vibrant and prospective image of China. "Fig. 3"
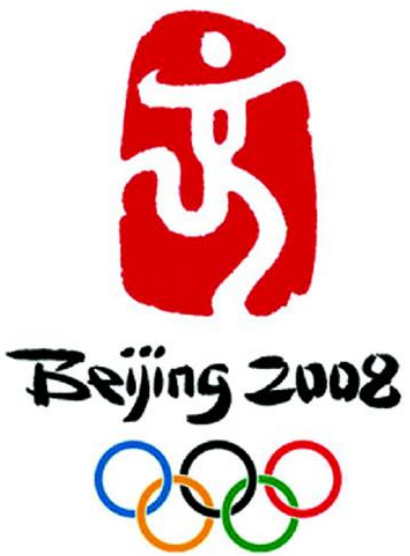

Fig. 3. The Logo of 2008 Beijing Olympic Games 
3) Harmony of Color Relationships: "Lun Yu " says "The gentleman aims at harmony, and not at uniformity. The mean man aims at uniformity, and not at harmony". "He" in "Harmony but not sameness" here means different things in harmony and unity ; "Tong" here refers to the simple sum or homogenization of the same thing. The ancients said, "Harmony generates and sameness stops vitality", which believes that only through coordinations of different components and factors, things can be formed. Things could not develop by repeat summing up the same thing. In the preQin era, "he" is a very important concept, it refers to unity of difference and diversity, which is different from "tong". Such as cooking, only by blending sour, sweet, bitter, spicy, salty all together to achieve a complete tastes of five and a flavor beyond the taste, can it be regarded as the finest cuisine; Such as music, only by mixing gong, shang, jiao, mei and yu all together to achieve a pentameter resonance and the sound beyond the instruments, can it be regarded as the finest melody : Conversely, if the salty lover keeping putting salt, the sour lover keep pouring vinegar, those who love gong exclude shang and jiao, those who love shang exclude yu and mei, the consequences would not be hard to imagine.

Triggered by the ancient yin and yang comes five colors interaction theory. The five colors are composed of oriental "green", southern "white", western "red", northern, "black" and "yellow" of the earth. Confucius's elaboration of colors are: "The superior man did not use a deep purple, or a puce color, in the ornaments of his dress." it means that the gentlemen would not use cloth of rosy deep blue or black with red color, nor would they use cloth of red or purple color for casual daily wear. These two colors are media colors which are considered inappropriate as official colors. Mauve is also not official color, so it is inappropriate even as color at home. For the interpretation of the painting, Confucius said, "More preparation may quicken the speed in doing work" which means that not just the ones with sweet appearance can be called beautiful, but people who understand and obey the etiquette is a real beauty. The pure and harmonious beauty in accordance with etiquette is the concept of color of Confucius.

Logo designing must be conducted under the principle of "Harmony but not sameness" delivering the beauty of harmony, by seeking change in unity and seeking unity in change. To achieve "Harmony but not sameness" in logo, is to achieve harmonious unity of different color, graphics, background and connotation by an overall design. China Mobile's new logo style with griping ties, is not only a good continuation of its brand image assets, but also makes the whole image more concise, dynamic, interoperable and smooth, and opens up extensible features of Internet. Light blue represents that China Mobile is stylish, friendly and smart, a piece of vivid green associate the brand image with innovation and social responsibility. "Fig. 4"

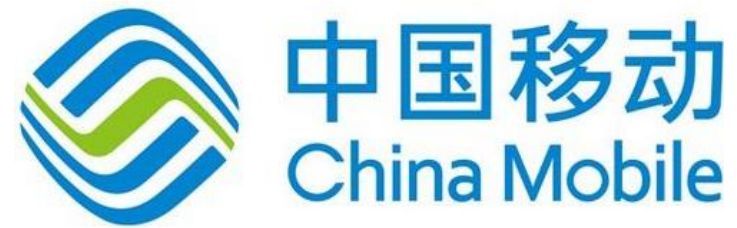

Fig. 4. The Logo of China Mobile

\section{HARMONIOUS THEORY IN THE USE OF LOGO DESIGNING}

Chinese philosophy of harmony is applied in the logo designing for Hoten Environment. Hoten Environment is a company dedicated to environmental governance and pursuit harmony between heaven, earth and human. Laozi said: "Human rules the earth, the earth rules the heaven, the heaven rules Tao, Tao rules nature." Taoist declare theory of nature, it believes that existence and development of all things are in accordance with the particular laws of the nature, thus, everything in the world should set nature as an example and follow nature. Human and nature unite as one, the main idea of Taoism, is the essence of the idea of Zhuangzi and is undertaking the idea of Laozi.

Hoten Environment logo is extracted from heaven, earth and human. "Earth" and "Human" under the "Heaven", "Earth" and "Human" interact and joint with harmony, the pursuit of harmonious relationship of "Human-human, human-objects, object-object, object-environment and human-environment", etc. In the logo, the outer circle represents "heaven", the graph in the middle represents "earth" and "human". The entire logo is a balance of righteousness, whereas never fail to deliver the sense of dynamic. The graph in the middle, looks like a Tai $\mathrm{Chi}$, is a symbol of human and nature live and grow in harmony. The blank in the graph looks like a river and a Chinese character "ren"(human), simple and vivid, representing freedom and natural life generating on a gentle and tolerant planet endlessly. The major colors of the logo are blue and green. The outer circle representing the heaven is of blue color to deliver hope, ideals, independent and smart. The inner circle representing the earth is of green color to bring people a sense of security, freedom, peace, happiness and vitality. Blue sky and green atmosphere complement each other and live in harmony. "Fig. 5"

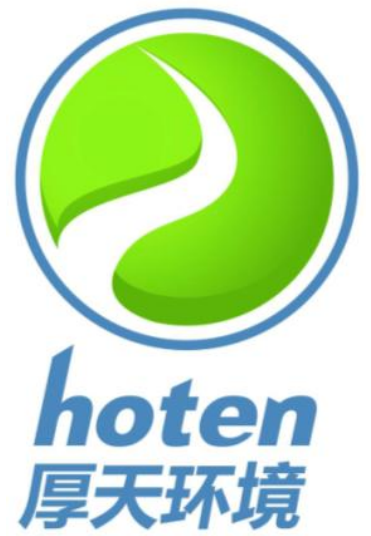

Fig. 5. The Logo of Hoten 
Chinese philosophy of harmony has a profound impact on the establishment of the Chinese modern design ideology and formation of aesthetic traits. To study the ideology of Chinese philosophy of harmony and to discuss its impact on logo designing helps to improve the quality of modern logo designing, and to form modern logo design with ethnic characteristics. Impact of Chinese philosophy of harmony in modern logo design ideas is not only reflected in worldview, but also reflected in the methodology, we should recall a lot of Chinese innovative ideas are derived from the sage's ideas and ways of thinking. Studying on their evolution, transformation and integration would allow us to gain revelations.

\section{REFERENCES}

[1] Written by (Shang Gu) Fu Xi, (Shang) Zhou Wenwang, (Chun Qiu) Confucius. Translated by Ling Yongfang. Zhou Yi [M]. China Pictorial Press, 2013

[2] (Chun Qiu) Depicted by Confucius, edited by Chen Tao. The Analects of Confucius [M]. People's Press of Yunnan, 2011.

[3] Zhang Jiemo. The aesthetics of Zen [M]. Peking University Press,2006.

[4] Li Yanzu. Visual express design of history and aesthetics[M]. People's University of China Press, 2000.

[5] Xun Shenglan. The source and flow -- the traditional culture and modern design [M]. Jiangxi Fine Arts Press, 2007.

[6] Duan Zemin. Modern logo design and Chinese traditional culture elements[J]. Method exploration. 